\title{
Sequential topologies and Dedekind finite sets*
}

\author{
Jindřich Zapletal \\ University of Florida
}

March 19, 2021

\begin{abstract}
It is consistent with ZF set theory that the Euclidean topology on $\mathbb{R}$ is not sequential, yet every infinite set of reals contains a countably infinite subset. This answers a question of Gutierres.
\end{abstract}

\section{Introduction}

This note deals with sequentiality of the Euclidean topology of the reals in choiceless context. To remind the reader of the standard definitions:

Definition 1.1. Let $X$ be a topological space.

1. A set $C \subset X$ is sequentially closed if every countable converging sequence of elements of $C$ converges to an element of $C$.

2. The space $X$ is sequential if every sequentially closed subset of $X$ is closed.

Under small fragments of the axiom of choice, such as the axiom of dependent choices (DC), it is easy to check the status of sequentiality of basic topological spaces. For example, all metric spaces are sequential, while $\omega_{1}+1$ with the order topology is not. Without the axiom of dependent choices, sequentiality becomes an issue even in the most basic contexts. Gutierres [1 showed that in ZF, sequentiality of the Euclidean topology of $\mathbb{R}$ has many equivalent restatements, and it implies that every infinite subset of $\mathbb{R}$ contains a countably infinite subset. He asked whether the opposite implication is provable in ZF. In this note, I show that this is not the case.

Theorem 1.2. It is consistent relative to an inaccessible cardinal that $Z F$ holds, every infinite subset of $\mathbb{R}$ contains a countably infinite subset, and the Euclidean topology of $\mathbb{R}$ is not sequential.

\footnotetext{
*2010 AMS subject classification 03E15, 03E25, 03E35.
} 
In the interests of brevity, the theorem understates the understanding of its associated model somewhat. In particular, in it every infinite set contains a countably infinite subset, and no uncountable Polish space is sequential. The inaccessible cardinal assumption is used only to make the construction fit under the umbrella of geometric set theory [3]; I do not know if it is necessary. It is also unclear if it is possible to distinguish between sequentiality of various uncountable Polish spaces; one rather egregious example is $\mathbb{R}$ and $\mathbb{R}^{2}$.

The paper uses standard set theoretic notation as in [2]; in matters of geometric set theory it follows [3].

\section{Proof of the main theorem}

The model for Theorem 1.2 is a forcing extension of the classical choiceless Solovay model by a certain Suslin poset which is designed in a straightforward way to add a witness for the failure of sequentiality of $\mathbb{R}$.

Definition 2.1. The poset $P$ consists of all pairs $p=\left\langle a_{p}, b_{p}\right\rangle$ where $a_{p} \subset[0,1]$ is a nowhere dense closed set and $b_{p} \subset[0,1]$ is a countable set disjoint from $a_{p}$. The ordering is defined by $q \leq p$ if $a_{p} \subset a_{q}$ and $b_{p} \subset b_{q}$. The $P$-name $\dot{A}$ is defined as the union of all first coordinates of conditions in the generic filter.

I make a couple of simple initial observations. First, conditions $p, q \in P$ are compatible if and only if $a_{p} \cap b_{q}=0$ and $a_{q} \cap b_{p}=0$. In such a case, there is a largest common lower bound of $p, q$, namely the condition $\left\langle a_{p} \cup a_{q}, b_{p} \cup b_{q}\right\rangle$. It follows immediately that the poset $P$ is Suslin. Since any point not in $b_{p}$ can be added to $a_{p}$ and any point not in $a_{p}$ can be added to $b_{p}$ obtaining a condition stronger than $p$, it also follows that $P$ forces the set $\dot{A} \subset[0,1]$ to be dense with dense complement; in particular, $\dot{A}$ is forced not to be closed.

The partial order $P$ is fairly useless in ZFC context. However, I will show that if $W$ is a choiceless Solovay model, then the $P$-extension of $W$ is a model for the theory required by Theorem 1.2 In order to do that, an analysis of its balanced virtual conditions as in [3] is necessary. This analysis takes place in ZFC. For every nowhere dense closed set $a \subset[0,1]$, let $\tau_{a}$ be the $\operatorname{Coll}(\omega,[0,1])$ name for the condition $\langle a,[0,1] \cap V \backslash a\rangle \in P$.

Theorem 2.2. In the poset $P$ :

1. for every nowhere dense closed set $a \subset[0,1]$, the pair $\left\langle\operatorname{Coll}(\omega,[0,1]), \tau_{a}\right\rangle$ is balanced;

2. for every balanced pair $\langle Q, \sigma\rangle$ there is a nowhere dense closed set $a \subset[0,1]$ such that the balanced pairs $\langle Q, \sigma\rangle$ and $\left\langle\operatorname{Coll}(\omega,[0,1]), \tau_{a}\right\rangle$ are equivalent;

3. distinct closed sets yield inequivalent balanced pairs.

In particular, the poset $P$ is balanced. 
Proof. For (1), suppose that $R_{0}, R_{1}$ are posets and $\sigma_{0}, \sigma_{1}$ are their respective names for conditions in $P$ which are stronger than $\langle a,[0,1] \cap V \backslash a\rangle$; I must show that $R_{0} \times R_{1} \Vdash \sigma_{0}, \sigma_{1}$ are compatible in $P$. Let $\sigma_{0}=\left\langle\dot{a}_{0}, \dot{b}_{0}\right\rangle$ and $\sigma_{1}=\left\langle\dot{a}_{1}, \dot{b}_{1}\right\rangle$.

Claim 2.3. $R_{0} \times R_{1} \Vdash \dot{a}_{0} \cap \dot{b}_{1}=0$ and $\dot{a}_{1} \cap \dot{b}_{0}=0$.

Proof. I will prove the latter conjunct; the proof of the former is symmetric. Suppose towards a contradiction that $\theta$ is an $R_{0}$-name for an element of $\dot{b}_{0}$ and $r_{0} \in R_{0}, r_{1} \in R_{1}$ are conditions which force in the product that $\theta \in \dot{a}_{1}$ holds. Let $M$ be a countable elementary submodel of a large structure containing $r_{0}, \theta$ in particular. Let $g \subset R_{0}$ be a filter generic over the model $M$ containing the condition $r_{0}$ and let $x=\theta / g$. Note that $R_{0} \Vdash \dot{b}_{0} \cap a=0$; so, it must be the case that $x \notin a$. Now, $R_{1} \Vdash \check{x} \notin \dot{a}_{1}$; so, there has to be a condition $r_{1}^{\prime} \leq r_{1}$ and a basic open neighborhood $O \subset[0,1]$ of $x$ such that $r_{1}^{\prime} \Vdash \dot{a}_{1} \cap O=0$. By the genericity of the filter $g$, there has to be a condition $r_{0}^{\prime} \leq r_{0}$ in $g$ such that $r_{0}^{\prime} \Vdash \theta \in O$. Then, the condition $\left\langle r_{0}^{\prime}, r_{1}^{\prime}\right\rangle$ forces in the product that $\theta \notin \dot{a}_{1}$ holds, contradicting the initial assumptions.

It follows immediately from the claim that the product forces $\left\langle\dot{a}_{0} \cup \dot{a}_{1}, \dot{b}_{0} \cup \dot{b}_{1}\right.$ to be a common lower bound of $\sigma_{0}, \sigma_{1}$. Item (1) follows.

For (2), let $\theta$ be the $Q$-name for the first coordinate of $\sigma$, and let $a=\{x \in$ $[0,1]: Q \Vdash \check{x} \in \theta$. I will show that $a \subset[0,1]$ is closed and the balanced pairs $\langle Q, \sigma\rangle$ and $\left\langle\operatorname{Coll}(\omega,[0,1]), \tau_{a}\right\rangle$ are equivalent. It is immediate that $a \subset[0,1]$ is closed and nowhere dense, since $Q \Vdash \theta$ is closed and nowhere dense. To conclude the proof, by [3, Proposition 5.2.6] it is enough to show that $Q \Vdash a \subset \theta$ and $([0,1] \cap V \backslash a) \cap \theta=0$ because then $\sigma$ and $\langle a,[0,1] \cap V \backslash a\rangle$ are forced to be compatible conditions in $P$.

To show that $Q \Vdash a \subset \theta$ holds, suppose that some condition $q$ forces the contrary. Then there has to be a basic open set $O \subset[0,1]$ such that $a \cap O \neq 0$ and $q \Vdash \theta \cap O=0$. Pick a point $x \in a \cap O \cap V$ and use the definition of the set $a$ to conclude that $Q \Vdash \check{x} \in \theta$. This immediately contradicts the assumption that $q \Vdash \theta \cap O=0$.

To show that $Q \Vdash([0,1] \cap V \backslash a) \cap \theta=0$ holds, suppose towards a contradiction that there is a point $x \in V \backslash a$ and a condition $q_{0} \in Q$ forcing $\check{x} \in \theta$. By the definition of the set $a$, there has to be a condition $q_{1} \in Q$ forcing $\check{x} \notin \theta$. Let $H_{0}, H_{1} \subset Q$ be mutually generic filters containing the conditions $q_{0}, q_{1}$ respectively, and let $p_{0}=\sigma / H_{0}$ and let $p_{1}$ be the condition obtained from $\sigma / H_{1}$ by adding the point $x$ to its second coordinate. It is clear that $p_{0}, p_{1}$ are conditions incompatible in $P$ as witnessed by the point $x$. This contradicts the initial balance assumption on the pair $\langle Q, \sigma\rangle$.

(3) is immediate. To get the last sentence, note that for every condition $p=\left\langle a_{p}, b_{p}\right\rangle$, the pair $\left\langle\operatorname{Coll}(\omega,[0,1]), \tau_{a}\right\rangle$ represents a balanced virtual condition stronger than $p$.

Corollary 2.4. Let $W$ be the choiceless Solovay model. Then $W \models P \Vdash \dot{A} \subset \mathbb{R}$ is sequentially closed. 
Proof. Suppose towards a contradiction that this fails. Recall that by 3 , Theorem 9.1.1] and the fact that the poset $P$ is balanced in ZFC, the $P$-extension of the model $W$ contains no new reals and no new $\omega$-sequences of reals. Thus, it must be the case that there is a condition $p \in P$ and an $\omega$-sequence $x$ for elements of $[0,1]$ converging to some $y$ such that $p \Vdash \operatorname{rng}(\check{x}) \subset \dot{A}$ and $\check{y} \notin \dot{A}$. The contradiction is achieved in two complementary cases. First, assume that $\operatorname{rng}(x) \subset a_{p}$. Then, by the closure of $a_{p}$, it is also true that $y \in a_{p}$ and $p \Vdash \check{y} \in \dot{A}$, contradicting the original assumptions. Second, assume that $\operatorname{rng}(x) \backslash a_{p}$ is nonempty, containing some point $z$. Consider the condition $q \leq p$ obtained from $p$ by adding $z$ to its second coordinate. Then $q \Vdash \operatorname{rng}(\check{x}) \not \subset \dot{A}$, again contradicting the initial assumptions.

As pointed out above, $\dot{A}$ is forced to be dense codense in $[0,1]$, so not closed. Thus, in the $P$-extension of the choiceless Solovay model, $\mathbb{R}$ is not a sequential space. All that remains to be proved is that in that extension, every infinite set of reals contains a countably infinite subset. This is in fact the main contribution of this paper. Theorem 1.2 is an immediate corollary.

Theorem 2.5. Let $W$ be the choiceless Solovay model. Then $W \models P \Vdash$ every infinite set of reals has a countably infinite subset.

Proof. Let $\kappa$ be an inaccessible cardinal, and let $W$ be the choiceless Solovay model derived from $\kappa$. Work in the model $W$. Let $\tau$ be a $P$-name for an infinite set of reals, and let $p \in P$ be a condition. I have to produce an injective function $\pi: \omega \rightarrow \mathbb{R}$ and a strengthening of the condition $p$ which forces $\operatorname{rng}(\pi) \subset \tau$. To this end, pick a parameter $z \in 2^{\omega}$ such that $\tau, p$ are both definable from $z$ and some additional parameters in the ground model. Let $V[K]$ be an intermediate generic extension obtained by a poset of cardinality smaller than $\kappa$ such that $z \in V[K]$. Work in $V[K]$.

Let $\bar{p}$ be the balanced virtual condition in $P$ associated with the set $a_{p}$ as in Theorem [2.2. If in the model $W, \bar{p} \Vdash \tau \subset V[K]$ holds, then $\bar{p} \Vdash \tau$ is countable, and the proof is complete. If this fails, then there must be a poset $Q$ of cardinality smaller than $\kappa$, a $Q$-name $\sigma$ for a condition stronger than $\bar{p}$ and $\theta$ and a $Q$-name $\theta$ for a real not in $V[K]$ such that $Q \Vdash \operatorname{Coll}(\omega,<\kappa) \Vdash \sigma \Vdash_{P} \theta \in \tau$. Move back to $W$.

First, let $H_{\omega} \subset Q$ be a filter generic over $V[K]$. Then, let $\left\langle H_{n}: n \in \omega\right\rangle$ be a sequence of filters on $Q$ which are pairwise mutually generic over $V[K]\left[H_{\omega}\right]$ and such that $\lim _{n} H_{n}=H_{\omega}$ in the usual topology of $\mathcal{P}(Q)$. This means that for every condition $q \in H_{\omega}$, for all but finitely many $n \in \omega q \in H_{n}$ holds. Write $p_{n}=\sigma / H_{n}, p_{n}=\left\langle a_{n}, b_{n}\right\rangle$, and $x_{n}=\theta / H_{n}$ for every index $n \in \omega$. It will be enough to show that the points $x_{n}$ for $n \in \omega$ are pairwise distinct and the conditions $p_{n}$ for $n \in \omega$ have a common lower bound in $P$, because then in the model $W$, that lower bound forces $\left\{x_{n}: n \in \omega\right\} \subset \tau$ as desired.

First of all, it is clear that the points $x_{n}$ for $n \in \omega$ are pairwise distinct, since if $n \neq m$ then $x_{n} \in V[K]\left[H_{n}\right] \backslash V[K]$ and $x_{m} \in V[K]\left[H_{m}\right] \backslash V[K]$, and the models $V[K]\left[H_{n}\right], V[K]\left[H_{m}\right]$ are mutually generic over $V[K]$. To see why the 
conditions $p_{n}$ for $n \in \omega$ have a common lower bound is more difficult. Write $p_{\omega}=\sigma / H_{\omega}, p_{\omega}=\left\langle a_{\omega}, b_{\omega}\right\rangle$.

Claim 2.6. $c=a_{\omega} \cup \bigcup_{n \in \omega} a_{n}$ is a closed nowhere dense subset of $[0,1]$.

Proof. For the closure I use the fact that $\lim _{n} H_{n}=H_{\omega}$. Suppose that $y \notin c$ is a point. Since $a_{\omega}$ is a closed set, there must be a basic open neighborhood $O_{\omega}$ of $y$ which is disjoint from $a_{\omega}$. There must be a condition $q \in H_{\omega}$ such that $q \Vdash O_{\omega}$ is disjoint from the first coordinate of the condition $\sigma \in P$. Find a number $n \in \omega$ such that for all $m>n, q \in H_{m}$ holds. Then for all $m>n$, $a_{m} \cap O_{\omega}=0$. For each index $m \leq n$, the set $a_{m}$ is closed and does not contain the point $y$, so there is a basic open neighborhood $O_{m}$ of $y$ disjoint from $a_{m}$. Clearly, $\bigcap_{m<n} O_{m} \cap O_{\omega}$ is an open neighborhood of $y$ disjoint from the set $c$.

To see that $c \subset[0,1]$ is nowhere dense, note that the set $[0,1] \cap V[K] \backslash a$ is dense and the set $c$ contains none of its points. Alternately, $c$ is a union of countably many closed nowhere dense sets, therefore meager and (as a closed set) nowhere dense.

Now, by the balance of the virtual condition $\bar{p}$, for all distinct indices $n, m \leq \omega$ it is the case that $p_{n}, p_{m}$ are compatible in $P$. This is to say that $a_{n} \cap b_{m}=0$ and $a_{m} \cap b_{n}=0$. It follows that $\bigcup_{n \leq \omega} a_{n}$ and $\bigcup_{n \in \omega} b_{n}$ are disjoint sets; they together form the requested common lower bound of all conditions $p_{n}$ for $n \in \omega$ or even $n \leq \omega$.

\section{References}

[1] Gonçalo Gutierres. Sequential topological conditions in $\mathbb{R}$ in the absence of the axiom of choice. Math. Log. Quart., 49:293-298, 2003.

[2] Thomas Jech. Set Theory. Springer Verlag, New York, 2002.

[3] Paul Larson and Jindřich Zapletal. Geometric set theory. AMS Surveys and Monographs. American Mathematical Society, Providence, 2020. 